\title{
Structural Investigation of the HIV-1 Reverse Transcriptase Initiation Complex by HDX, SAXS, Cryo-EM, and X-ray Crystallography
}

\author{
Chelsy Chesterman ${ }^{1}$, Steven Tuske ${ }^{1}$, Jie Zheng ${ }^{2}$, Youngmin Jeon ${ }^{3}$, Cheng Zhang ${ }^{3}$, William \\ Cantara $^{4}$, Karin Musier-Forsyth ${ }^{4}$, Patrick R. Griffin ${ }^{2}$, Dmitry Lyumkis ${ }^{3}$, Eddy Arnold ${ }^{1}$ \\ ${ }^{1}$ Center for Advanced Biotechnology and Medicine, Rutgers University \\ 2Department of Molecular Therapeutics, The Scripps Research Institute Florida \\ ${ }^{3}$ Laboratory of Genetics and Helmsley Center for Genomic Medicine, Salk Institute for Biological Studies \\ ${ }^{4}$ Department of Chemistry and Biochemistry, Ohio State University
}

Over the past 30 years, a concerted effort has been made to understand the full enzymatic and virological cycle of HIV-1 reverse transcriptase (RT) through structural biology, biochemistry, molecular biology, and virology techniques. RT plays an essential role in HIV replication, and is the target of nearly half of the drugs used to treat HIV infection. A critical stage of reverse transcription that has not been visualized is the initial complex, in which a portion of the viral genomic RNA binds to provide the template (primer-binding site) and reverse transcription is primed by tRNA $\mathrm{Lys}^{3}$ from the host cell. This reverse transcription initiation complex is relatively challenging to produce in sufficient quantity and quality for high-resolution structure determination.

We are using a range of hybrid methods in a multi-laboratory collaboration to determine the most stable variants of this complex to use for high-resolution structural biology techniques such as X-ray crystallography and cryo-electron microscopy (cryo-EM), as well as to obtain information on which portions of the RNA and HIV-1 RT are in proximity. Among the key factors is choosing truncations of viral RNA and tRNA that combine to form more stable in vitro complexes. A multi-stage workflow has been used to screen multiple truncations by hybrid techniques including size-exclusion chromatography coupled with small angle X-ray scattering and hydrogen-deuterium exchange coupled with mass spectrometry. These techniques allow us to map the interactions between RT and the VRNA/tRNA complexes and select purified, nonaggregated samples that are enriched in the complete complex. This has informed sample preparation for cryo-EM, and allowed us to generate initial cryo-EM structures for the reverse transcription initiation complex. This presentation will include our multi-technique workflow and the resulting structures of the reverse transcription initiation complex. 